\title{
A sustainable food support for non-breastfed infants: implementation and acceptability within a WHO mother-to- child HIV transmission prevention trial in Burkina Faso
}

\author{
Cécile Cames ${ }^{1, *}+$, Claire Mouquet-Rivier ${ }^{2}$, Tahirou Traoré ${ }^{3}$, Kossiwavi A Ayassou $^{4}$, \\ Claire Kabore $^{3}$, Olivier Bruyeron ${ }^{5}$ and Kirsten B Simondon ${ }^{1}$ \\ 'UMR145, IRD/UM1, Montpellier, France: ${ }^{2} U M R 204$, IRD/UM1/UM2/SupAgro, Montpellier, France: \\ ${ }^{3}$ GRET, Ouagadougou, Burkina Faso: ${ }^{4}$ Centre Muraz, Bobo-Dioulasso, Burkina Faso: \\ ${ }^{5}$ GRET, Nogent-sur-Marne, France
}

Submitted 18 June 2009: Accepted 20 January 2010: First published online 1 March 2010

\begin{abstract}
Objective: To provide HIV-positive mothers who opted for exclusive breastfeeding or formula feeding from birth to 6 months postpartum as a means of prevention of mother-to-child transmission (PMTCT) of HIV with a sustainable infant food support programme (FSP) from 6 to 12 months postpartum. We describe the implementation and assessment of this pilot initiative.

Design: The FSP included a 6-month provision of locally produced infant fortified mix (IFM; $418 \mathrm{~kJ} / 100 \mathrm{~g}$ of gruel) for non-breastfed infants coupled with infantfeeding and psychosocial counselling and support. Acceptability and feasibility were assessed in a subsample of sixty-eight mother-infant pairs.

Setting: The FSP was developed in collaboration with local partners to support participants in a PMTCT prevention study. Formula was provided for free from 0 to 6 months postpartum. Cessation by 6 months was recommended for breastfeeding mothers.

Results: The FSP was positively received and greatly encouraged breastfeeding mothers to cease by 6 months. As recommended, most infants were given milk as an additional replacement food, mainly formula subsidised by safety networks. Among daily IFM consumers, feeding practices were satisfactory overall; however, the IFM was shared within the family by more than one-third of the mothers. Cessation of IFM consumption was observed among twenty-two infants, seventeen of whom were fed milk and five neither of these.

Conclusions: Without any food support most mothers would have been unable to provide appropriate replacement feeding. The food security of non-breastfed infants urgently needs to be addressed in HIV PMTCT programmes. Our findings on a simple cost-effective pioneer intervention provide an important foundation for this process.
\end{abstract}

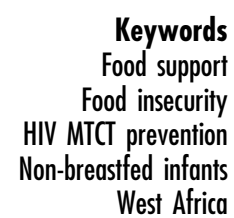

Keywords

Food support HIV MTCT prevention West Africa
HIV-infected mothers can prevent postnatal mother-tochild transmission (MTCT) of HIV by avoiding breastfeeding from birth when formula feeding is acceptable, feasible, affordable, sustainable and safe, or by exclusively breastfeeding for the first 6 months of the infant's life and initiating rapid weaning if adequate replacement infant food is available ${ }^{(1)}$. Research conducted among HIV-exposed breastfed infants in Malawi suggested that infections occurring after 6 months of age accounted for more than $85 \%$ of late postnatal infections ${ }^{(2)}$. Early

† Correspondence address: Centre IRD, UMR145, BP 64501, 34394 Montpellier Cedex, France. breastfeeding cessation could thus greatly reduce postnatal infection rate but its implementation remains challenging. Indeed, in Zambia, abrupt breastfeeding cessation around 5 months postpartum has been associated with increased infant mortality and reduced HIVfree survival rates in a large MTCT prevention study ${ }^{(3)}$.

Early breastfeeding cessation is not a common practice in sub-Saharan Africa and is mainly a consequence of maternal death ${ }^{(4)}$. Among breastfed infants, breast milk contributes approximately $50 \%$ and $30 \%$ of energy needs from 6 to 12 months and 12 to 24 months, respectively. It is therefore extremely difficult to cover the nutritional needs of non-breastfed infants. To fill the gap in terms of 
knowledge and practices, guiding principles for feeding non-breastfed children 6-24 months of age have been made available ${ }^{(5)}$. Non-breastfed infants should be given whole animal milk (or formula, if available) and nutritive replacement foods divided into three to five meals per $\mathrm{d}$ according to the energy density of the replacement food. Translating these guidelines into practice is particularly challenging in Burkina Faso, one of the world's poorest countries, where complementary feeding practices are inadequate in terms of the quality and quantity of the food given, as well as the timing of introduction of semisolid and solid foods (being either too early or too late). Prolonged breastfeeding is an entrenched practice: $98 \%$ and $81 \%$ of children are breastfed at birth and at 23 months of age, respectively. Most children under 2 years of age are given traditional gruels early as main and regular complementary food ${ }^{(6,7)}$. These inexpensive gruels are prepared daily from fermented pearl millet and sold in ready-to-eat form in the streets and markets. Their fluid consistency results in poor nutritional quality, with an energy density below $182 \mathrm{~kJ}(44 \mathrm{kcal}) / 100 \mathrm{~g}^{(7)}$. In terms of the national infant food supply, most locally produced infant mixes do not satisfy the recommended nutrient composition for complementary foods ${ }^{(8)}$, and imported ones are not affordable for most of the population (C MouquetRivier and $\mathrm{S}$ Hervé, unpublished results). The suboptimal complementary food pattern may contribute to the high prevalence of malnutrition observed among Burkinabe children (stunting is above $40 \%$ among 6-35-month-olds and wasting is above $30 \%$ among 6-24-month-olds) ${ }^{(6)}$.

The Kesho Bora Study (KBS) on MTCT prevention in Bobo-Dioulasso recruited HIV-infected pregnant women who were assigned to various regimens and durations of antiretroviral (ARV) prophylaxis or treatment according to their stage of HIV disease. Those with CD4 cell counts below 200 or above 500 at inclusion in late pregnancy were included in observational cohorts, while women with CD4 counts from 200 to 500 were randomised to a trial comparing the impact of standard short-term pre- and perinatal ARV prevention of MTCT to highly active ARV treatment from late pregnancy to 6 months postpartum ${ }^{(9)}$.

The KBS protocol included sustained pre- and postpartum infant-feeding counselling, recommending either exclusive breastfeeding and rapid weaning around 6 months postpartum or exclusive formula feeding from birth (6 months' free formula was provided), a strategy that is consistent with the current Burkinabe MTCT prevention policy $^{(10)}$. If maternal adherence to infant feeding recommendations was good, we expected that most infants would have a 'non-breastfed' status from 6 months of age, whatever their initial feeding mode, and thus a greater subsequent risk of malnutrition and death than their breastfed counterparts.

The first objective of this operational research was to implement an adequate and sustainable food support programme (FSP) for HIV-infected mothers who decided to avoid or shorten breastfeeding, as a means of prevention of postnatal transmission. Second, we aimed to assess this FSP with regard to its acceptability and feasibility among mothers and infants in terms of feeding practices. Ultimately, this operational research was conceived to prepare the groundwork for future support networks and stakeholders' large-scale interventions if proved successful in this context.

An assessment of infants' total food consumption (24 h recall and $1 \mathrm{~d}$ direct food weighing) and its relation to infant nutritional status and growth was also conducted, and will be published separately.

\section{Methods}

The initiative was launched in 2005 with food technologists involved in the NUTRIFASO project based in Ouagadougou as a collaboration between the Research Institute for Development (IRD) and the Research and Technology Exchange Group (GRET). The main objective of NUTRIFASO is to sustainably improve the nutritional status of infants and women of childbearing age by optimising feeding practices. This is translated into actions to promote access to high-quality complementary food based on local products and healthy practices.

The aim was to provide an infant fortified mix (IFM) based on locally produced and commonly consumed food ingredients (and/or local alternative foods) that conforms to cultural food habits and allows for safe and easy handling. In the present study, the term 'IFM' refers to the generic or dry product while 'IFM gruel' refers to the edible form, i.e. mixed with water and cooked.

\section{Composition and production of the infant fortified mix}

The IFM formulation was conceived to ideally meet the nutrient and energy needs of infants from 6 to 12 months of age $^{(11)}$ on the condition that gruel was given in combination with whole powdered milk or formula, which were the two main types of milk mothers were expected to use. The energy content was $1733 \mathrm{~kJ}$ ( $414 \mathrm{kcal}) / 100 \mathrm{~g}$ of raw product, $20.5 \%$ of which came from lipids, $13.0 \%$ from proteins and $66.5 \%$ from carbohydrates. The nutritional value of local ingredients (maize, soyabean, groundnut and sugar) was enhanced by adding a vitamin and mineral commercial premix (Fortitech, Denmark). Alpha-amylases (Novo, Denmark) were introduced to the IFM to increase the energy density of gruels prepared at a consistency suitable for consumption by infants. Thus, $100 \mathrm{~g}$ of fluid IFM gruel provided at least $418 \mathrm{~kJ}(100 \mathrm{kcal})$. Since it was recommended that mothers provide $450 \mathrm{ml}$ of milk per $\mathrm{d}, 100 \mathrm{~g}$ of IFM (i.e. 400 to $450 \mathrm{~g}$ of gruel to be given in two to four meals) was sufficient to cover all daily nutrient needs.

The IFM was produced by a local manufacturer, a partner of NUTRIFASO that received training in good 


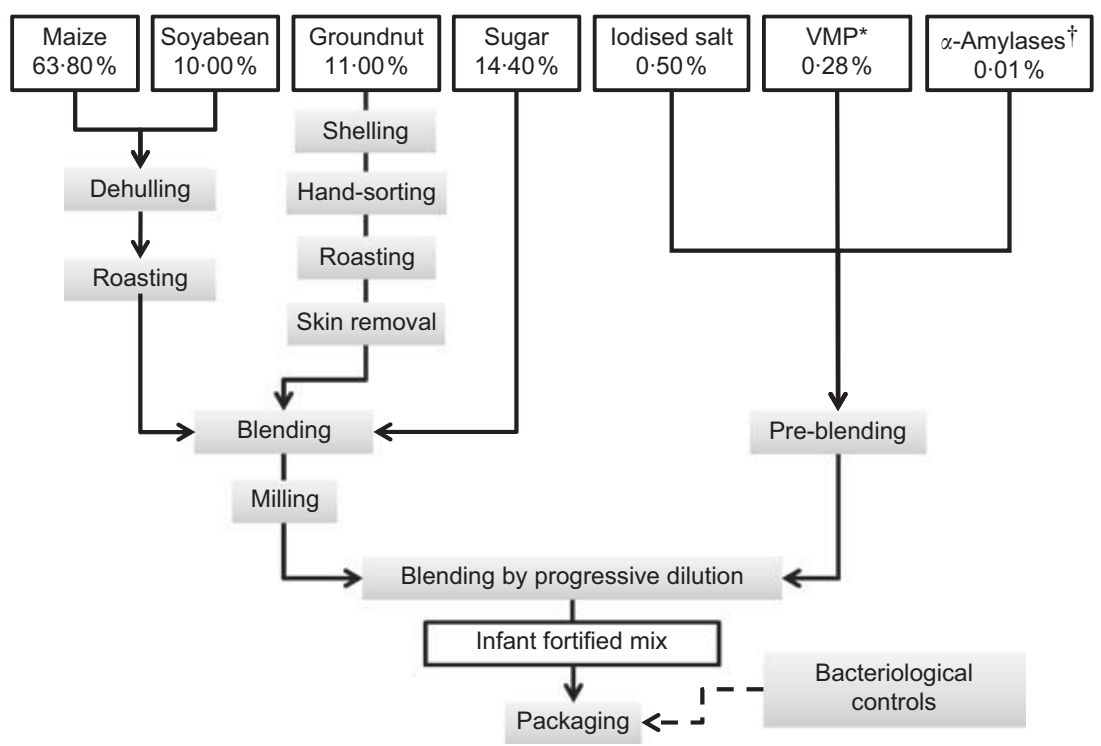

Fig. 1 Composition and processing of the infant-fortified mix (raw content; *vitamins and minerals premix, Fortitech Burk1, Gadstrup, Denmark; †BAN 800 mg Novo SA, Bagsvaerd, Denmark)

Table 1 Comparison of infant fortified mix (IFM) with other local or imported processed infant mixes, according to their retail price and energy density (ED), as specified on the packaging by the manufacturer and as measured in experimental conditions

\begin{tabular}{|c|c|c|c|c|c|}
\hline \multirow[b]{2}{*}{ Infant mix } & \multirow[b]{2}{*}{ Retail price/kg $(€)$} & \multicolumn{2}{|c|}{ ED: specified $/ 100 \mathrm{~g}$ of gruel } & \multicolumn{2}{|c|}{ ED: experimental/100 g of gruel* } \\
\hline & & kJ & kcal & kJ & kcal \\
\hline Recommended minimum EDt & & 351 & 84 & 351 & 84 \\
\hline \multicolumn{6}{|l|}{ Local products } \\
\hline IFM ‘Biiga Zoom' & $2 \cdot 1$ & 418 & 100 & 418 & 100 \\
\hline Vitacasui & $2 \cdot 3$ & 426 & 102 & 263 & 63 \\
\hline Vitaline & 3.9 & 389 & 93 & 259 & 62 \\
\hline Den Mugu & $2 \cdot 8$ & 217 & 52 & 125 & 30 \\
\hline \multicolumn{6}{|l|}{ Imported products } \\
\hline Phosphatine & $9 \cdot 8$ & 376 & 90 & 389 & 93 \\
\hline Bledine & $10 \cdot 4$ & 447 & 107 & 414 & 99 \\
\hline Cérélac & $7 \cdot 7$ & 472 & 113 & 460 & 110 \\
\hline
\end{tabular}

*Measured using a Bostwick flow parameter $=120 \mathrm{~mm} / 30 \mathrm{~s}$, which corresponds to an appropriate consistency of gruels for young children (C Mouquet-Rivier and S Hervé, unpublished results).

tLutter and Dewey ${ }^{(8)}$.

production practices. The production process included steps to clean, dehull and roast each of the raw materials to reduce microbial contamination (Fig. 1). Each batch also underwent bacteriological testing by the National Public Health Laboratory in Ouagadougou. Analyses reported that the microbiological quality of the IFM complied with the French norms for cooking infant's mix foods. Bags of $120 \mathrm{~g}$ and $500 \mathrm{~g}$ were delivered in double plastic hygienic packaging with no apparent brand mark. Nutritional facts and instructions for safe handling and preparation were provided on the packaging. Although the food was provided free of charge within the KBS, affordability was included in the requirements. The actual retail price was similar to or lower than that of good-quality local products, i.e. $2 \cdot 1 € / \mathrm{kg}$ (Table 1)

\section{Food support programme components}

The FSP was introduced among KBS participants at the end of 2005. As per protocol, all mothers received a $3.0 \mathrm{~kg}$ monthly allowance at 6 months postpartum (at breastfeeding cessation or at the end of formula provision) until their infants reached 12 months of age, while they were requested to purchase milk on their own. At the first distribution, mothers were invited to come with their utensils so that the counsellor could show them the quantities of water and flour to be mixed together, according to the infant's age. Demonstrations of IFM gruel, whole powdered milk and formula preparations were performed, followed by a retrodemonstration by the mother. Moreover, mothers were provided with specific counselling on replacement food and related feeding practices at each monthly visit to the KBS site. 
Throughout the study follow-up, mothers were also encouraged to disclose their HIV status to partners and significant others and seek support from community HIV networks.

\section{Food support programme assessment and etbical concerns}

An observation study on FSP perceptions and mothers' feeding practices was conducted on a subsample of participants between March and June 2007. At that time, 200 mothers were enrolled and followed up in the KBS; seventy-seven had an infant between 6 and 12 months of age. Only seventy were fulfilling the inclusion criterion (i.e. having a non-breastfed infant) for IFM provision and were given individual information about the study's objectives and procedures. All gave written consent and participated in the observation study. Since the survey revealed that two infants were actually still breastfed, the final sample numbered sixty-eight mother-infant pairs. Mothers were visited at home by trained staff purposively recruited for the study. An observation session of IFM preparation and feeding was held unbeknownst to the mothers. Infants' usual consumption of IFM and milk and mothers' evaluations of IFM characteristics and their experience of the FSP were collected using a semistructured questionnaire.

\section{Results}

The mean age of infants was 9.2 (SD 1.6) months; fifty-six infants were previously breastfed and twelve were previously formula fed. All mothers stated that they initiated IFM gruel feeding at first distribution. However, among sixty-eight infants, forty-six had been given IFM gruel and milk at least once on the day of the survey and were described by their mothers as regular consumers. The usual daily number of feeds ranged from one to four for both IFM gruel and milk. Infants who did not consume IFM gruel on the day of the survey were described by their mother as occasional or non-consumers, divided into regular milk consumers ( $n 17$ ), usually receiving one to four milk feeds per $\mathrm{d}$, and milk non-consumers ( $n 5)$. Milk prepared by the mothers ( $n$ 63) was formula ( $77 \%$ ) or whole powdered milk. In most cases $(71 \%)$, milk was subsidised by HIV support networks or the national PMTCT programme. Alternatively, mothers bought milk from shops. A total of $46 \%$ of the mothers were illiterate, $54 \%$ had no means of income, $46 \%$ had not disclosed their HIV status to their partner and $76 \%$ had no access to safe drinking water. These proportions were found to be similar whether or not the child was an IFM consumer.

\section{Handling, preparation and feeding of the infant fortified mix}

Observations of the first feed were performed for the forty-six regular consumers to assess the mothers' compliance
Table 2 Infant feeding practices and behaviours of Burkinabe HIVinfected mothers participating in the food support programme with regard to the infant fortified mix (IFM)

\begin{tabular}{lrc}
\hline & \multicolumn{2}{c}{$\begin{array}{c}\text { Mothers of regular } \\
\text { consumers of IFM }\end{array}$} \\
\cline { 2 - 3 } & $n 46$ & $\%$ \\
\hline IFM preparation & & \\
Hygiene & & \\
$\quad$ Adequate storage & 43 & 93 \\
$\quad$ Utensils washed with water and soap & 35 & 76 \\
$\quad$ Cleaning of preparation area & 29 & 63 \\
Type of water used & & \\
$\quad$ Indoor tap & 8 & 17 \\
$\quad$ Drums from community tap & 38 & 83 \\
Preparation & & \\
$\quad$ Adequate amount of ingredients in mix & 42 & 91 \\
$\quad$ Adequate boiling time (at least 5 min) & 30 & 65 \\
IFM feeding & & \\
$\quad$ Hand washing & 3 & 6 \\
$\quad$ Active feeding & 23 & 50 \\
Feeding mode & & \\
$\quad$ Cup-fed & 10 & 22 \\
$\quad$ Spoon-fed & 25 & 54 \\
$\quad$ Bottle-fed & 6 & 13 \\
$\quad$ Mixed-fed \\
IFM allowance shared within the family
\end{tabular}

*Relevant for multiparous mothers ( $n$ 29).

with recommended practices in terms of storage, preparation and feeding of the IFM gruel (Table 2).

The IFM was stored in satisfactory hygienic conditions in almost all households, but safe preparation areas and access to a safe drinking water source were not widespread. Mothers did not delegate IFM preparation ( $n$ 45) or feeding ( $n 44)$; many stated that this was too important to be entrusted to someone else. Most of them followed the mixing recommendations; however, few followed the cooking instructions. The preparation mode was judged as 'difficult' by eight mothers, mainly due to the need to mix and cook ingredients.

With regard to IFM gruel feeding, the main concerns proved to be low compliance to routine hygienic practices, like washing hands, and basic recommendations in terms of active feeding. Only half of the mothers ( $n$ 23) persevered in feeding their infants when faced with rejection, and at least six mothers used a bottle to feed the infant the gruel, although this was strongly discouraged by counsellors. Surprisingly, this was the case for former breastfeeders, while former formula feeders fed their infant with a cup or a spoon.

\section{Mothers' perceptions of the food support programme and its components}

The sensory characteristics of the IFM gruel greatly differ from what people are used to. The most popular local millet gruel has a very sour taste (a result of lactic acid fermentation), and large quantities of sugar are added just before consumption. In addition, it presents a grey colour 
Table 3 Evaluation by Burkinabe HIV-infected mothers of the characteristics of the infant fortified mix (IFM) gruel

\begin{tabular}{|c|c|c|c|c|c|}
\hline \multirow[b]{2}{*}{ Sensory qualities } & \multicolumn{4}{|c|}{ Appreciation ( $n$ 68) } & \multirow[b]{2}{*}{ Main comments } \\
\hline & Excellent & Good & Medium & Bad & \\
\hline Appearance & 63 & 4 & 1 & 0 & No residual dust or lumps in gruel - good packaging \\
\hline Colour & 12 & 54 & 2 & 0 & Light yellow colour of maize mixed with milk \\
\hline Texture & 5 & 54 & $\overline{9}$ & 0 & Smooth and creamy consistency \\
\hline Smell & 19 & 44 & 3 & 1 & Milky cookie smell \\
\hline Flavour & 25 & 45 & 0 & 0 & Sweetened milk taste \\
\hline Nutritional quality & Better/equivalent & Worse & No opinion & & \\
\hline Compared to local mix & 65 & 1 & 2 & & No need to add anything to enrich the gruel \\
\hline Compared to imported mix & 27 & 1 & 40 & & \\
\hline
\end{tabular}

and may contain lumps depending on whether millet flour granules are added during cooking. However, a majority of mothers endorsed the IFM's sensory properties whether their infants were consuming it regularly or not, and perceived the IFM as a high-quality food regarding both nutritional and hygienic characteristics (Table 3). Surprisingly, they associated the IFM with a milk product even though this ingredient is absent from the recipe.

Women were very positive about receiving a food support, which greatly encouraged them in their infantfeeding choices. Most of the mothers who had been previously breastfeeding their infants stated that the prospect of receiving a food support at breastfeeding cessation greatly encouraged them to choose the exclusive breastfeeding option at birth and, later, to initiate breastfeeding cessation.

A majority ( $n$ 64) expressed satisfaction with the nutrition counselling provided and showed sound knowledge of quantity ( $n$ 66) and quality ( $n 55$ ) of meals to be given to infants. During the course of the KBS, the IFM turned out to be an adequate home-based rehabilitation food for women experiencing weight loss and for infants with growth failure at any time during follow-up (unpublished data). In our sample, more than $25 \%$ of the mothers were prescribed an IFM allowance in late pregnancy, and $7 \%$ thought the need would arise for their own health but were not eligible.

With regard to IFM distribution, among mothers of regular consumers ( $n$ 46), seventeen considered the monthly quantity insufficient, and thirty described the allowance duration as too short (the remainder either thought the allowance would last 18 months or were unaware of its duration). However, one-third of these forty-six mothers reported that they shared the allowance with their family. Among these forty-six mothers, thirtyone stated that they were also giving small quantities of other foods (traditional gruel, family meal and snacks) every day. The most frequently cited reason was to accustom the infant to different food tastes, thus probably anticipating the end of the FSP. Most mothers ( $n$ 40) felt that their infant looked healthier since starting the IFM. This perception was based on their observation of regular weight gain each month at the study clinic, shiny skin and good motor development. Ultimately, forty-three of the women affirmed their capacity to purchase the IFM in the future at a medium price of $0.56 €$ per bag ( $500 \mathrm{~g}$ ), which is half the retail price.

Despite this positive perception of the FSP components, twenty-two infants were no longer consuming the IFM gruel at the time of the survey. Most of their mothers ( $n$ 18) stated that they faced temporary or permanent rejection associated with an infant's episode of illness. Cross-referencing socio-economic data with counsellors' accounts showed that the five mothers who gave neither IFM nor milk were particularly vulnerable. Only one had shared her HIV status with her partner, four were illiterate, none had access to safe drinking water and all had experienced serious psychosocial difficulties with their kin. Such unfavourable conditions limited mothers' ability to utilise food support within the KBS, seek food support outside the KBS or purchase adequate infant food, and certainly affected their self-efficacy and autonomy in infant-feeding practices.

\section{Discussion and conclusion}

Perceived acceptability of the IFM and the FSP among the study participants was high. Most of the mothers genuinely cared about the quality of the FSP components and had a good knowledge of an infant's nutritional needs due to in-depth and repeated counselling. The feasibility of the different components of the FSP (diet composition, feeding and hygiene practices) was quite satisfactory for two-thirds of the mother-infant pairs and the IFM and milk played an important role in these infants' diet.

However, one-third of infants were no longer consuming the IFM gruel, mainly as a consequence of an episode of illness. Common illnesses are known to decrease complementary food consumption among breastfed infants ${ }^{(12)}$. The high energy density of the IFM gruel and decreased infant appetite may explain some cases of slowdown in consumption or rejection, particularly if the traditional thin gruel was already introduced in 
the diet. Alternatively, mothers might associate the occurrence of an episode of illness with the IFM gruel (particularly in cases of diarrhoea) and intentionally reduce the portion or withdraw it from the diet and instead feed the infant with the traditional fluid millet gruel, which can be given as a drink. Discussions with study clinic staff in charge of infant feeding counselling suggested that poor feeding practices, the mothers' lack of time and, in some cases, low motivation might partly explain these unsuccessful experiences. Among mothers of IFM non-consumers, none were regularly providing an appropriate alternative infant food, and five of them were not even able to obtain milk.

These results suggest that without any food support (in and outside the KBS) most mothers would have been unable to provide appropriate replacement foods for their infants, but this does not exclude the possibility that they would have ceased breastfeeding early. The authors of a study conducted in Zimbabwe reported that HIVpositive mothers in resource-constrained settings may be so motivated to protect their child from HIV that they stop breastfeeding early even when they cannot provide an adequate replacement diet ${ }^{(13)}$. Such findings highlight the dilemma an informed HIV-infected mother faces at the time of infant feeding decisions when she is forced to choose between two competing risks: exposure to malnutrition or exposure to HIV infection.

The present study also highlighted the particular constraints posed by the FSP that some mothers were not willing or able to deal with. IFM and milk preparation is more time and energy consuming than ready-to-eat millet gruel, which does not require any specific equipment. Depending on circumstances, this resulted in poor adherence to the FSP or in a lack of compliance to hygiene and feeding recommendations. As a consequence, both nutritional and hygienic quality of food given and quantities could be affected. Decreased incomes and poor health and psychosocial conditions due to their HIV infection are known to negatively impact people's behaviour regarding family feeding practices ${ }^{(14)}$. Psychosocial support and enhanced nutritional education are thus major components to be included in FSP to ensure optimal acceptability and benefits among vulnerable populations.

All members of HIV-affected families potentially need a nutritional support, as a consequence of decreased household productivity and the high expenditure on care ${ }^{(15)}$. Providing an 'infant-targeted' food aid is likely to lead to sharing of the allowance within the family to the detriment of the non-breastfed infant. To overcome such expected and understandable leakages, food rations provided by most household-based support programmes are usually larger than needed. The present FSP was implemented within a PMTCT study offering a comprehensive package of health and support-related services. Therefore, we limited the food allowances to non-breastfed infants from 6 to 12 months of age and undernourished women and children up to 24 months postpartum (i.e. the end of follow-up). This FSP assessment showed that IFM allowance leakages were quite common among the study population and probably under-reported. This result illustrates the importance of targeting all pre-school children in the household, instead of merely non-breastfed infants if resources are available. Options for FSP extension up to 18 or 24 months postpartum (i.e. the average duration of breastfeeding) should also be considered in future projects.

This intervention suggested that this highly nutrientdense food, which is easy to swallow and digest, could adequately enhance the diet of HIV-infected mothers who experience troubles with appetite and weight loss. Acceptability is expected to be high in communities where most people usually eat cereal-based gruel for breakfast $^{(7)}$. Provision of such a nutritive food complement to HIV-infected pregnant and lactating women is worth consideration as a routine PMTCT intervention, given the impact of maternal nutritional status on HIV disease progression ${ }^{(16)}$, vertical transmission and pregnancy outcomes ${ }^{(17,18)}$.

Thus far, the common response to the food insecurity of HIV-affected households has often relied on community safety nets, with their limited sustainability of financial and human resources. Moreover, to join a safety network requires that the beneficiaries disclose their HIV status, and this may be a strong barrier to seeking support for many HIV-positive women ${ }^{(19)}$.

To the best of our knowledge, no operational research data have been published so far on the implementation and effectiveness of FSP for HIV-exposed non-breastfed children in sub-Saharan Africa. Recent publications show that most short-term food support interventions are designed to specifically target HIV-infected adults ${ }^{(20,21)}$. Intervention based on ready-to-use therapeutic food (RUTF) proved to be acceptable and effective in home management of malnutrition in HIV-infected and uninfected children ${ }^{(22,23)}$. Within this operational research programme in Malawi, it has been shown that the RUTF spread form could be safely and easily produced in most developing settings ${ }^{(24)}$. In this context, the production cost of RUTF was approximately US\$ $2 \cdot 60 / \mathrm{kg}$ between 2002 and 2005, which is quite similar to the cost of the IFM between 2005 and $2009(2 \cdot 1 € / \mathrm{kg})$ in Burkina Faso. However, RUTF used as a long-term nutritive input in the daily diet of HIV-exposed non-breastfed children and vulnerable groups (HIV-infected or not) still needs to be assessed with regard to acceptability, efficacy and costeffectiveness criteria. Preliminary results from an ongoing PMTCT study in Malawi have suggested a high level of acceptance of RUTF used as a post-weaning 5-month food support ${ }^{(25)}$.

We developed this project in collaboration with the local private sector and national and international institutions, involving food and health scientists and production and marketing experts. Such a partnership is essential 
in ensuring the cost-effectiveness of food fortification programmes ${ }^{(26)}$. We did not conduct a comprehensive cost-effectiveness assessment including all services related to IFM distribution (i.e. storage, nutritional counselling), but the net cost of a 6-month IFM provision was $37 \cdot 8 €$ per infant in our study. Cost savings can be realised by multiplying production units and developing alternative IFM recipes that are less costly to produce, which is a main component of the NUTRIFASO project objectives. All of the ingredients of the recipe are grown or produced in Burkina Faso, except for the vitamin and mineral premix and amylases that must still be imported from Europe. The cost of any IFM, even if locally produced and if cost savings could be expected in the future, is beyond the reach of the poorest families in which children are the most vulnerable to malnutrition. Scaling-up of such an intervention would thus require support from governmental or non-governmental agencies for funding and the integration of public health sectors and communities as full partners in food support management, promotion and distribution.

Complex interactions among HIV/AIDS, nutritional status and food insecurity are well known, and their determinants and implications have been fully documented. In 2006, the United Nations took a major step forward when it recognised the urgent need to integrate food support as part of a comprehensive response to HIV and $\operatorname{AIDS}^{(27)}$. International concern over the health and survival of HIV-exposed non-breastfed infants is far more recent. Nevertheless, this new hotbed of food insecurity raises a growing interest and was highlighted at the last WHO Technical Consultation on Nutrition and HIV held in Burkina Faso ${ }^{(28)}$. However, successful nutrition integration in PMTCT programmes is far from easy, and raises many programmatic issues that will need a pragmatic approach and the aggregate involvement of all stakeholders to put statements into practice. In Burkina Faso, almost 15000 pregnant women were HIV-infected according to the lowest national estimates in 2004. Consequently, more than 1300 paediatric infections were expected per year ${ }^{(29)}$. The current National Four-Year Plan for PMTCT, which aims to scale up efforts made during the previous period mainly in terms of health services coverage and pregnant women's adherence to PMTCT services, does not mention any specific food support programming ${ }^{(29)}$.

This operational research sheds some light on how food aid can be included in current national guidelines on infant feeding and HIV. Development and dissemination of more research experiences, particularly in the context of PMTCT programmes, are needed to foster advocacy for scaling-up such successful approaches ${ }^{(30)}$. We hope that this initiative will inform and stimulate support networks, public health sectors and stakeholders in designing interventions to alleviate the food insecurity burden on HIV-affected families. If particular attention is paid to specific foods and nutrition security for HIV-exposed (and also HIV-infected) infants, scaling-up of nutritional support to these children in Burkina Faso would certainly be facilitated, less stigmatising and probably more efficient if embedded in a wide-ranging effort to improve the population's access to appropriate infant food. However, the particularly critical situation of infants with no or limited breastfeeding and of their HIV-positive mothers should be acknowledged as it may not be able to wait for a national programme to be implemented.

Our experience shows that efforts made to provide locally fortified food coupled with nutritional education and monitoring allow for making the best use of local resources and potentially reaching all members of AIDS-affected households with an expected low risk of HIV-related stigma. We thus recommend that such a fortified food be formally tested for efficacy in sustaining infant growth and improving the nutritional status of undernourished children and adults. The forthcoming challenge will be to bridge the gap between short-term targeted support to individuals and longer-term efficient food security programming for vulnerable groups. Our initiative of a simple, local and cost-effective pioneer intervention provides an important foundation for this process.

\section{Acknowledgements}

Source of funding: The study was funded by the Agence Nationale de Recherches sur le SIDA (ANRS), Paris, France and is part of the ANRS 1271/Kesho Bora cohort study. Authors' contributions: C.C. conceived and implemented the operational research, conducted data analysis and drafted the first version of the manuscript. C.M.R., O.B., K.B.S. and T.T. contributed to the conception of the research, interpretation of the results and writing of the manuscript. K.A.A. and C.K. monitored the research and revised the manuscript. Acknowledgements: The authors are grateful to the Kesho Bora study and the NUTRIFASO project, and especially to Roger Lankoandé for his skilful contribution to the research implementation. They particularly thank Biego Samssonna, flour manufacturer, for his fruitful collaboration in producing the IFM; Maïmouna Sawadogo, Cécile Koala and Fanny Cassart, who collected the data; and to all the women who took part in the observational study for their kind collaboration. Conflict of interest: The authors have no conflict of interest related to the present study.

\section{References}

1. World Health Organization (2007) HIV and Infant Feeding. Update based on the Technical Consultation held on behalf of the Inter-agency Task Team (IATT) on Prevention of HIV Infection in Pregnant Women, Mothers and their Infants. Geneva: WHO. 
2. Taha TE, Hoover DR, Kumwenda NI et al. (2007) Late postnatal transmission of HIV-1 and associated factors. J Infect Dis 196, 10-14.

3. Kuhn L, Aldrovandi GM, Sinkala M et al. (2008) Effects of early, abrupt weaning on HIV-free survival of children in Zambia. N Engl J Med 359, 130-141.

4. Mane NB, Simondon KB, Diallo A et al. (2006) Early breastfeeding cessation in rural Senegal: causes, modes, and consequences. Am J Public Health 96, 139-144.

5. World Health Organization (2005) Guiding Principles for Feeding Non-breastfed Children 6-24 Months of Age. Geneva: WHO.

6. Institut National de la Statistique et de la Démographie \& ORC Macro (2004) Demographic and Health Survey in Burkina Faso 2003. Calverton, MD: ORC Macro \& INSD.

7. Mouquet-Rivier C, Icard-Verniere C, Guyot JP et al. (2008) Consumption pattern, biochemical composition and nutritional value of fermented pearl millet gruels in Burkina Faso. Int J Food Sci Nutr 59, 716-729.

8. Lutter CK \& Dewey KG (2003) Proposed nutrient composition for fortified complementary foods. J Nutr 133, 3011S-3020S

9. de Vincenzi I, Kesho Bora Study Group (2009) Tripleantiretroviral (ARV) prophylaxis during pregnancy and breastfeeding compared to short-ARV prophylaxis to prevent mother-to-child transmission of HIV-1 (MTCT): the Kesho Bora randomized controlled clinical trial in five sites in Burkina Faso, Kenya and South Africa. Presented at Fifth IAS Conference on HIV Pathogenesis Treatment and Prevention, Cape Town, 19-22 July 2009 (abstract).

10. Ministère de la Santé du Burkina Faso (2006) Directives Nationales pour la Mise en Euvre du Programme National de Prévention de la Transmission Mère-Enfant du VIH. Ouagadougou: Ministère de la Santé.

11. Dewey KG \& Brown KH (2003) Update on technical issues concerning complementary feeding of young children in developing countries and implications for intervention programs. Food Nutr Bull 24, 5-28.

12. Brown KH, Stallings RY, de Kanashiro HC et al. (1990) Effects of common illnesses on infants' energy intakes from breast milk and other foods during longitudinal community-based studies in Huascar (Lima), Peru. Am J Clin Nutr 52, 1005-1013.

13. Lunney KM, Jenkins AL, Tavengwa NV et al. (2008) HIVpositive poor women may stop breast-feeding early to protect their infants from HIV infection although available replacement diets are grossly inadequate. J Nutr 138, 351-357.

14. Alkenbrack Batteh SE, Forsythe S, Martin G et al. (2008) Confirming the impact of HIV/AIDS epidemics on household vulnerability in Asia: the case of Cambodia. Aids 22, Suppl. 1, S103-S111.

15. Bukusuba J, Kikafunda JK \& Whitehead RG (2007) Food security status in households of people living with HIV/ AIDS (PLWHA) in a Ugandan urban setting. Br J Nutr 98, 211-217.
16. Fawzi WW, Msamanga GI, Spiegelman D et al. (2004) A randomized trial of multivitamin supplements and HIV disease progression and mortality. $N$ Engl J Med 351, 23-32.

17. Villamor E, Saathoff E, Msamanga G et al. (2005) Wasting during pregnancy increases the risk of mother-to-child HIV-1 transmission. I Acquir Immune Defic Syndr $\mathbf{3 8}$, 622-626.

18. Mehta S, Manji KP, Young AM et al. (2008) Nutritional indicators of adverse pregnancy outcomes and mother-tochild transmission of HIV among HIV-infected women. Am J Clin Nutr 87, 1639-1649.

19. Foster $G$ (2007) Under the radar: community safety nets for AIDS-affected households in sub-Saharan Africa. AIDS Care 19, Suppl. 1, S54-S63.

20. Byron E, Gillespie S \& Nangami M (2008) Integrating nutrition security with treatment of people living with HIV: lessons from Kenya. Food Nutr Bull 29, 87-97.

21. Cantrell RA, Sinkala M, Megazinni K et al. (2008) A pilot study of food supplementation to improve adherence to antiretroviral therapy among food-insecure adults in Lusaka, Zambia. J Acquir Immune Defic Syndr 49, 190-195.

22. Ciliberto MA, Sandige H, Ndekha MJ et al. (2005) Comparison of home-based therapy with ready-to-use therapeutic food with standard therapy in the treatment of malnourished Malawian children: a controlled, clinical effectiveness trial. Am J Clin Nutr 81, 864-870.

23. Ndekha MJ, Manary MJ, Ashorn P et al. (2005) Home-based therapy with ready-to-use therapeutic food is of benefit to malnourished, HIV-infected Malawian children. Acta Paediatr 94, 222-225.

24. Manary MJ (2006) Local production and provision of readyto-use therapeutic food (RUTF) spread for the treatment of severe childhood malnutrition. Food Nutr Bull 27, S83-S89.

25. Thomas R, Tembo M, Bramson B et al. (2006) Evaluation of mothers' experiences using a ready-to-use therapeutic food to aid in early breasfeeding cessation of infants of HIVinfected mothers: the BAN study in Lilongwe, Malawi. Presented at Sixteenth International AIDS Conference, Toronto, 13-18 August 2006 (abstract).

26. Mannar MG \& van Ameringen M (2003) Role of publicprivate partnership in micronutrient food fortification. Food Nutr Bull 24, S151-S154.

27. United Nations General Assembly Special Session Dedicated to HIV/AIDS (2006) Declaration of Commitment. New York: UNGASS

28. World Health Organization (2008) Regional Consultation on Nutrition and HIV/AIDS for French Speaking Countries. Preliminary Report. Geneva: WHO.

29. Ministère de la Santé du Burkina Faso (2006) Programme National de Prévention de la Transmission Mère-Enfant du VIH 2006-2010. Ouagadougou: Ministère de la Santé.

30. Kadiyala S \& Gillespie S (2004) Rethinking food aid to fight AIDS. Food Nutr Bull 25, 271-282. 